\title{
Post-traumatic Stress Disorder, Race/Ethnicity, and Coronary Artery Disease Among Older Patients with Depression
}

\author{
Denise C. Cooper • Ranak B. Trivedi • Karin M. Nelson • \\ Gayle E. Reiber • Kristine A. Beaver • \\ Evercita C. Eugenio • Vincent S. Fan
}

Received: 23 January 2014 / Revised: 23 April 2014 / Accepted: 30 April 2014 / Published online: 23 May 2014

(C) W. Montague Cobb-NMA Health Institute 2014

\begin{abstract}
Objectives Post-traumatic stress disorder (PTSD) and depression are both associated with coronary artery disease (CAD) in older adults. It is unclear whether PTSD is associated with CAD among older patients with depression, particularly ethnic/racial minority patients. We examined how PTSD relates to $\mathrm{CAD}$ in older depressed patients and how this relationship differed by race/ethnicity.

Methods Medical records were used to identify White, Black, Hispanic, Asian, and American Indian/Alaskan Native
\end{abstract}

D. C. Cooper ' K. M. Nelson ' G. E. Reiber ' K. A. Beaver

E. C. Eugenio • V. S. Fan

Center of Innovation for Veteran-Centered and Value-Driven Care,

Health Services Research and Development (HSR\&D), Veteran

Affairs (VA) Puget Sound Health Care System, Seattle, WA, USA

D. C. Cooper · G. E. Reiber

Department of Health Services, University of Washington, Seattle,

WA, USA

R. B. Trivedi

Center for Innovation to Implementation, VA Palo Alto Health Care

System, Palo Alto, CA, USA

R. B. Trivedi

Department of Psychiatry and Behavioral Sciences, Stanford

University, Palo Alto, CA, USA

K. M. Nelson • V. S. Fan

Department of Medicine, University of Washington, Seattle,

WA, USA

D. C. Cooper $(\square)$

HSR\&D, VA Puget Sound Health Care System, 1100 Olive Way,

Suite 1400, Seattle, WA 98101, USA

e-mail: cooped2@uw.edu

D. C. Cooper

e-mail: denise.cooper3@va.gov patients ( $n=24,719$; ages 60-96 years) with chronic depression. Adjusted logistic regressions were conducted to assess the cross-sectional association between PTSD and CAD in each racial/ethnic group.

Results Among depressed Black patients, PTSD was associated with a $29 \%$ increase in odds for CAD [adjusted odds ratio $(\mathrm{AOR})=1.29 ; 95 \%$ confidence interval $(\mathrm{CI}), 1.01,1.66]$. Conversely, PTSD was associated with decreased odds for CAD among Whites (AOR $=0.90 ; 95 \% \mathrm{CI}$ : 0.84, 0.98) and Hispanics $(\mathrm{AOR}=0.65 ; 95 \% \mathrm{CI}$ : 0.44, 0.94). PTSD was not significantly associated with CAD in Asian or American Indian/Alaskan Native patients.

Conclusion The relationship between PTSD and CAD varied among depressed patients according to race/ethnicity. Older Black adults with depression may be at increased risk for CAD if they have comorbid PTSD.

Keywords Post-traumatic stress disorder · Coronary artery disease · African Americans · Hispanics · Asians · American Indians

\section{Introduction}

Post-traumatic stress disorder (PTSD) has been linked to increased risk for coronary artery disease (CAD) [1-3]. PTSD is found among an estimated $6 \%$ of the general population and among $11.5 \%$ of all Veterans receiving care at Veterans Affairs (VA) primary clinics [3, 4]. Data suggest older Black, Hispanic, Asian, and American Indian/Alaskan Native (AI/AN) Veterans may have higher rates of PTSD than their White counterparts [5-8]. In addition to Black Veterans and other racial/ethnic minority Veterans having higher rates of PTSD, they often have elevated risk for CAD [9, 10]. However, research is scarce on the relationship between PTSD and CAD among racial/ethnic minority populations. 
PTSD often co-occurs with depression [11-13], which also has been linked to increased risk for CAD $[14,15]$. Data from a study of current mental disorders among US Veterans reported that $82 \%$ of Veterans with PTSD also had depression [16]. Despite this high psychiatric comorbidity, little is known about whether PTSD raises the risk of CAD among individuals with depression.

Research on CAD with PTSD and comorbid depression has largely been limited to a few studies of survey data that found PTSD was associated with self-reported angina after adjustment for depressed mood [3, 17]. Studies using the clinical data of Veteran populations with depression have reported that PTSD was associated with significant coronary stenosis [18] and was not associated with risk for acute cardiac events [19], but neither study provided race/ethnicity-specific data. The purpose of the current study was to examine how PTSD relates to diagnoses of CAD among older White, Black, Hispanic, Asian, and American Indian/Alaskan Native patients with depression.

\section{Methods}

\section{Participants}

This cohort of patients with chronic depression $(n=24,719)$ included White, Black, Hispanic, Asian, and American Indian/Alaskan Native older Veterans (60-96 years old) from a larger study of patients seen in VA outpatient care for depression treatment during fiscal years 2009 and 2010 [20]. Patients were deemed chronically depressed based on electronic medical records showing a recent visit for depression (ICD-9 codes: 296.2x, 296.3x, 298.0, 300.4, 301.12, 309.0, $309.1,309.28,311)$ and evidence of depression treatment in the 4 months prior to their most recent depression visit based on chart review. As previously described, ${ }^{20}$ these data were derived from the Department of Veterans Affairs (VA) External Peer Review Program (EPRP), which selected patients at random from inpatient and outpatient settings to be included in a standardized review of abstracted electronic medical records to assess VA quality of care [21]. Specific groups (e.g., women) were over-sampled for quality purposes. Data from the EPRP were linked to inpatient and outpatient administrative data [22], beneficiary and vital status files, and data from the VA Corporate Data Warehouse.

Due to the design of the larger study examining 6 months of depression treatment [20], the current sample does not include patients with bipolar disorder or schizophrenia, patients who died within the 6 months after their most recent depression diagnosis, or patients from the Inpatient Spinal Cord Injury Unit (due to receiving majority of their care outside the VA). Psychiatric and medical diagnoses were derived from ICD-9 codes found for $\geq 1$ inpatient visit or $\geq 2$ outpatient visits. VA administrative records for mental health and cardiology diagnoses show good reliability when compared with medical records [19, 23]. The Institutional Review Board of the VA Puget Sound Health Care System approved the study.

\section{Measures}

\section{Outcome Variable: Coronary Artery Disease}

Data on active CAD diagnoses were derived from ICD-9 codes (411.xx, 413.xx, 414.0x, 414.2, 414.8, 414.9, 429.2). Of interest were acute and subacute forms of ischemic heart disease, angina pectoris, other forms of chronic ischemic heart disease, and arteriosclerotic cardiovascular disease.

\section{Independent Variable: PTSD}

PTSD Diagnosis of PTSD was based on ICD-9 code 309.81.

\section{Covariates}

Socio-demographic Factors Data were collected about patients' age, sex, and marital status (married vs. unmarried).

Cardiovascular Risk Factors and Medical Comorbidities Outpatient and inpatient data were used to identify hypertension, diabetes, hyperlipidemia, obesity, and other medical comorbidities. The Charlson-Deyo Comorbidity Index [24] was modified to remove illnesses that overlapped with other study variables in order to capture other medical comorbidities. Chart abstraction provided data on smoking (never smoked, former smoker, current smoker) status and alcohol use via AUDIT-C scores, such that higher AUDIT-C scores indicate higher self-reported alcohol use/ misuse $[25,26]$.

Treatment and Utilization Factors Due to the potential influence of medical and psychiatric service utilization, which often differs by race/ethnicity [27], data were obtained on the prior year's number of visits to primary care and mental health clinics. Data also were collected on the number of outpatient psychotherapy sessions and inpatient psychiatric hospitalizations during the 6 months following the most recent depression diagnosis to provide information suggesting the extent of recent psychiatric difficulties and level of treatment [28].

Data Analysis

Descriptive analyses were conducted to characterize the sample. Chi-square tests and analysis of variance were used to examine racial/ethnic differences in unadjusted variables. A 
series of logistic regressions were conducted to assess the association of PTSD with CAD among each racial/ethnic group. PTSD was entered in the logistic regressions after a standard set of covariates, which were selected based on prior literature [12, 19, 20, 27-29] and showed significant correlations with the variables of interest. These covariates were age, sex, marital status, hypertension, diabetes, hyperlipidemia, obesity, other medical comorbidities, smoking, AUDIT-C scores (alcohol use), prior primary care and mental health clinic visits, and recent treatment in inpatient and outpatient (psychotherapy sessions) care.

All variables in the analyses were categorical, with the exception of the continuous measures of age, primary care visits, psychotherapy sessions, and AUDIT-C scores. To reduce the substantial positive skew in the variables for psychotherapy sessions and AUDIT-C scores, these variables were $\log 10$ transformed.

\section{Results}

\section{Sample Characteristics}

Overall, data on sample characteristics (Table 1) suggested that racial/ethnic minority patients were significantly different from White patients and that the Black, Hispanic, Asian, and American Indian/Alaskan Native patients were often notably different from each other. CAD varied significantly by race/ethnicity $(p<0.001)$. Unadjusted data indicate that CAD diagnoses were most prevalent among Whites $(27 \%)$ and least prevalent among Blacks (17\%). Conversely, PTSD was more common among all racial/ethnic minorities than Whites $(p<0.001)$, with PTSD found in $40 \%$ of Whites, $48 \%$ of Hispanics, $50 \%$ of Asians, $56 \%$ of American Indians/Alaskan Natives, and $58 \%$ of Blacks. Compared to their White, Hispanic, and Asian counterparts, Blacks were younger $\left(p^{\prime} \mathbf{s}<0.001\right)$ and less likely to be married $(p ' s \leq 0.001)$, similar to American Indians/Alaskan Natives. Hispanics had the highest proportion of married Veterans $(61 \%)$.

Unadjusted data on cardiovascular risk factors and other comorbidities indicated that each racial/ethnic group had the highest prevalence of at least one cardiovascular risk factor. Having hypertension and $\geq 3$ other Charlson-Deyo comorbidities was most common among Blacks (68 \% and $10 \%$, respectively). Hispanics had the highest rate of diabetes (47\%), while Whites and Asians had the highest prevalence of hyperlipidemia (51\%). Whites also had the highest rate of diagnosed obesity (13\%). Current smoking was highest among American Indians/Alaskan Natives (27\%), Blacks (27\%), and Whites (24\%), but among these groups, American Indians/Alaskan Natives had the greatest proportion of patients that had quit smoking (13\%). Compared to Whites and Hispanics, Blacks had higher prior utilization of mental health clinics $(\geq 11$ visits) ( $p$ 's $<0.001)$. Blacks also were more likely to undergo a psychiatric hospitalization than their White counterparts $(p<0.001)$.

\section{Logistic Regressions of PTSD on CAD by Race/Ethnicity}

Results for the race/ethnicity-stratified logistic regressions of PTSD on CAD among older adults with depression are shown in Table 2 and described below.

\section{Whites}

PTSD was significantly related to lower risk of CAD among Whites (adjusted odds ratio $[\mathrm{AOR}]=0.90 ; 95 \%$ confidence interval [CI],: $0.84,0.98)$. With the exception of obesity, covariates representing medical and socio-demographic risk factors for $\mathrm{CAD}$ (i.e., hypertension, diabetes, hyperlipidemia, smoking, older age, male sex) were all associated with increased odds of CAD. Surprisingly, odds of CAD were increased among Whites who were married $(\mathrm{AOR}=1.12 ; 95 \%$ CI: 1.04,1.20), but were decreased if they reported greater levels of alcohol use (AOR $=0.74 ; 95 \%$ CI: $0.62,0.89)$ or had $\geq 1$ psychiatric hospitalization $(\mathrm{AOR}=0.73 ; 95 \% \mathrm{CI}$ : 0.61 , $0.88)$.

\section{Blacks}

Among Blacks, PTSD was associated with a $29 \%$ increase in the odds of having a $\mathrm{CAD}$ diagnosis $(\mathrm{AOR}=1.29 ; 95 \% \mathrm{CI}$ : $1.01,1.66)$. Fewer covariates were associated with $\mathrm{CAD}$ among these patients than their White counterparts. Like their White counterparts, Black patients who utilized mental health clinics in the prior year $\geq 11$ times had significantly decreased odds of CAD (AOR $=0.46 ; 95 \%$ CI: 0.30, 0.69).

\section{Hispanics}

Like their White counterparts, Hispanics had lower odds of CAD if they had PTSD (AOR: 0.65; $95 \%$ CI: 0.44, 0.94). Older age $(\mathrm{AOR}=1.03 ; 95 \% \mathrm{CI}: 1.01,1.06)$ and having $\geq 3$ Charlson-Deyo comorbidities (AOR $=3.73 ; 95 \%$ CI: 2.18 , 6.38) were associated with significantly increased odds of $\mathrm{CAD}$. Hispanic patients with a higher number of primary care visits in the prior year had decreased odds of CAD (AOR= 0.953; 95 \% CI: 0.910, 0.998).

\section{Asians}

The association between PTSD and CAD did not reach statistical significance $(p=0.07)$ among Asians, though the estimate is similar to the finding in Hispanics ( $\mathrm{AOR}=0.60 ; 95 \%$ CI: $0.35,1.05)$. Few significant associations were found between covariates and CAD. Similar to their White 
Table 1 Sample characteristics by race/ethnicity $(n=24,719)$

\begin{tabular}{|c|c|c|c|c|c|c|}
\hline Variable & $\begin{array}{l}\text { White } \\
(n=20,297)\end{array}$ & $\begin{array}{l}\text { Black } \\
(n=2,629)\end{array}$ & $\begin{array}{l}\text { Hispanic } \\
(n=986)\end{array}$ & $\begin{array}{l}\text { Asian } \\
(n=461)\end{array}$ & $\begin{array}{l}\text { American Indian/ } \\
\text { Alaskan Native } \\
(n=346)\end{array}$ & $p$ \\
\hline Post-traumatic stress disorder $(\%)$ & 40.4 & 57.5 & 48.1 & 50.1 & 55.5 & $<0.001$ \\
\hline Coronary artery disease $(\%)$ & 27.0 & 17.0 & 22.2 & 21.3 & 22.8 & $<0.001$ \\
\hline \multicolumn{7}{|l|}{ Socio-demographic factors } \\
\hline Age (years) $[M(\mathrm{SD})]$ & $67.0(7.6)$ & $64.8(5.8)$ & $66.2(7.1)$ & $66.6(7.2)$ & $64.8(5.8)$ & $<0.001$ \\
\hline Sex: $\operatorname{men}(\%)$ & 90.2 & 89.8 & 94.0 & 89.4 & 85.8 & $<0.001$ \\
\hline Married (\%) & 57.3 & 47.1 & 61.0 & 56.8 & 49.7 & $<0.001$ \\
\hline \multicolumn{7}{|l|}{ Cardiovascular risk factors and comorbidities } \\
\hline Hypertension (\%) & 55.2 & 67.6 & 61.6 & 59.0 & 50.0 & $<0.001$ \\
\hline Diabetes $(\%)$ & 37.9 & 42.8 & 47.2 & 45.6 & 43.4 & $<0.001$ \\
\hline Hyperlipidemia (\%) & 51.3 & 45.9 & 48.6 & 51.0 & 44.2 & $<0.001$ \\
\hline Obesity (\%) & 13.1 & 11.0 & 10.5 & 11.1 & 11.0 & 0.002 \\
\hline Other comorbidities (Charlson-Deyo): 0 (\%) & 63.1 & 60.9 & 65.2 & 64.4 & 62.7 & $<0.001$ \\
\hline $1(\%)$ & 16.0 & 13.0 & 12.0 & 18.0 & 17.1 & \\
\hline $2(\%)$ & 13.6 & 16.1 & 14.0 & 10.2 & 14.2 & \\
\hline$\geq 3(\%)$ & 7.3 & 10.0 & 8.8 & 7.4 & 6.1 & \\
\hline Smoking status: Never smoked (\%) & 64.2 & 62.0 & 75.7 & 71.2 & 60.1 & $<0.001\}$ \\
\hline Former smoker $(\%)$ & 11.4 & 11.3 & 9.5 & 9.3 & 13.3 & \\
\hline Current smoker $(\%)$ & 24.3 & 26.7 & 14.8 & 19.5 & 26.6 & \\
\hline Alcohol use (AUDIT-C scores) [M (SD)] & 0.57 & 0.51 & 0.54 & 0.48 & 0.53 & 0.006 \\
\hline \multicolumn{7}{|l|}{ Treatment and utilization factors } \\
\hline Prior mental health clinic visits $0(\%)$ & 26.6 & 15.8 & 19.7 & 17.7 & 21.7 & \\
\hline $1-2(\%)$ & 18.7 & 18.8 & 19.7 & 19.5 & 12.4 & \\
\hline $\begin{array}{l}3-4(\%) \\
5-10(\%)\end{array}$ & 17.7 & 17.4 & 19.4 & $\begin{array}{l}16.9 \\
23.8\end{array}$ & $\begin{array}{l}21.1 \\
237\end{array}$ & $<0.001\}$ \\
\hline$\geq 11(\%)$ & 16.4 & 25.0 & 18.4 & 22.1 & 21.1 & \\
\hline Prior primary care visits [M (SD)] & $4.7(4.6)$ & $4.9(4.5)$ & $5.0(3.9)$ & $4.9(4.7)$ & $5.2(5.2)$ & 0.003 \\
\hline$\geq 1$ psychiatric hospitalization (\%) & 4.2 & 6.4 & 5.9 & 4.3 & 6.9 & $<0.001$ \\
\hline Psychotherapy sessions [M (SD)] & $2.1(4.9)$ & $3.1(7.1)$ & $2.5(6.1)$ & $2.6(5.3)$ & $2.5(5.4)$ & $<0.001$ \\
\hline
\end{tabular}

counterparts, married Asians had higher odds of CAD $(\mathrm{AOR}=2.10 ; 95 \% \mathrm{CI}: 1.23,3.60)$.

\section{American Indians/Alaskan Natives}

This group did not exhibit a significant association between PTSD and CAD. Few associations were found between CAD and the covariates, other than male sex and having $\geq 3$ other medical comorbidities. In addition, odds of CAD increased in this group if they had hypertension $(\mathrm{AOR}=1.93 ; 95 \% \mathrm{CI}$ : $1.01,3.69)$.

\section{Discussion}

In this sample of older patients with chronic depression, the association of PTSD with CAD varied by race/ethnicity. PTSD was associated with a higher likelihood of CAD, but only among Blacks. These older Black patients with depression had a $29 \%$ increase in the odds of having a CAD diagnosis if they had PTSD. The opposite was true for depressed White and Hispanic patients whose odds for CAD were $10 \%$ and $35 \%$ lower, respectively, if they had comorbid PTSD. Asian and American Indian/Alaskan Native patients with depression showed no association between PTSD and CAD. To our knowledge, this is first study to provide race/ethnicity-specific data on diagnosed CAD in relation to PTSD and relevant correlates for older Black, Hispanic, Asian, or American Indian/Alaskan Native adults with depression.

Overall, unadjusted data showed that CAD diagnoses were more prevalent among Whites than Blacks. This is similar to reports of Blacks having less coronary obstruction than Whites, despite having higher mortality from CAD [30]. However, our results indicated that these Black depressed patients lose their lower CAD advantage if they also have 
Table 2 Logistic regressions of PTSD on CAD by race/ethnicity: adjusted odds ratios with confidence intervals

\begin{tabular}{|c|c|c|c|c|c|c|}
\hline \multicolumn{2}{|l|}{ Variable } & White & Black & Hispanic & Asian & $\begin{array}{l}\text { American Indian/ } \\
\text { Alaskan Native }\end{array}$ \\
\hline \multicolumn{2}{|l|}{ Age } & $1.02(1.02,1.03)^{* * *}$ & $1.04(1.02,1.06)^{* * *}$ & $1.03(1.01,1.06)^{*}$ & $1.03(0.99,1.07)$ & $1.01(0.95,1.06)$ \\
\hline \multicolumn{2}{|l|}{ Male Sex } & $3.51(2.98,4.15)^{* * *}$ & $2.95(1.70,5.09)^{* * *}$ & $1.74(0.73,4.14)$ & $4.43(1.00,19.63)$ & $6.20(1.65,23.20) * *$ \\
\hline \multicolumn{2}{|l|}{ Married } & $1.12(1.04,1.20) * *$ & $0.97(0.77,1.22)$ & $1.30(0.92,1.85)$ & $2.14(1.25,3.66)^{* *}$ & $0.93(0.51,1.70)$ \\
\hline \multicolumn{2}{|l|}{ Hypertension } & $1.20(1.12,1.30)^{* * *}$ & $1.24(0.94,1.62)$ & $1.08(0.75,1.58)$ & $0.71(0.40,1.27)$ & $1.93(1.01,3.69) *$ \\
\hline \multicolumn{2}{|l|}{ Diabetes } & $1.32(1.23,1.42)^{* * *}$ & $1.28(1.02,1.60)^{*}$ & $1.25(0.88,1.76)$ & $1.61(0.95,2.73)$ & $1.44(0.80,2.61)$ \\
\hline \multicolumn{2}{|l|}{ Hyperlipidemia } & $1.53(1.42,1.64) * * *$ & $1.68(1.33,2.11)^{* * *}$ & $1.22(0.85,1.73)$ & $1.48(0.85,2.56)$ & $1.70(0.92,3.13)$ \\
\hline \multicolumn{2}{|l|}{ Obesity } & $1.08(0.98,1.20)$ & $0.74(0.52,1.07)$ & $1.23(0.72,2.10)$ & $0.80(0.34,1.85)$ & $0.99(0.40,2.43)$ \\
\hline \multicolumn{2}{|l|}{ Other Comorbidities: 0} & Reference & Reference & Reference & Reference & Reference \\
\hline \multicolumn{2}{|l|}{1} & $3.95(3.63,4.31)^{* * *}$ & $3.89(2.92,5.20)^{* * *}$ & $5.59(3.57,8.75)^{* * *}$ & $2.93(1.62,5.33)^{* * *}$ & $2.65(1.28,5.52)^{*}$ \\
\hline \multicolumn{2}{|l|}{2} & $1.73(1.57,1.90)^{* * *}$ & $1.41(1.03,1.93)^{*}$ & $2.37(1.52,3.71)^{* * *}$ & $1.54(0.68,3.48)$ & $1.71(0.74,3.95)$ \\
\hline \multicolumn{2}{|l|}{$\geq 3$} & $3.61(3.21,4.06)^{* * *}$ & $3.19(2.31,4.41)^{* * *}$ & $3.73(2.18,6.38)^{* * *}$ & $3.67(1.55,8.67)^{* *}$ & $6.03(1.93,18.89)^{* *}$ \\
\hline \multicolumn{2}{|l|}{ Smoking: Never smoked } & Reference & Reference & Reference & Reference & Reference \\
\hline \multicolumn{2}{|l|}{ Former smoker } & $1.19(1.07,1.32)^{* *}$ & $1.73(1.25,2.39)^{* *}$ & $0.60(0.32,1.15)$ & $1.26(0.54,2.96)$ & $1.55(0.67,3.63)$ \\
\hline \multicolumn{2}{|l|}{ Current smoker } & $1.10(1.01,1.20)^{*}$ & $1.22(0.93,1.60)$ & $1.41(0.89,2.24)$ & $0.98(0.49,1.96)$ & $0.97(0.48,1.98)$ \\
\hline \multicolumn{2}{|l|}{ Alcohol use } & $0.74(0.62,0.89)^{* *}$ & $0.56(0.30,1.05)$ & $1.67(0.72,3.91)$ & $1.13(0.26,4.96)$ & $0.43(0.09,2.03)$ \\
\hline \multicolumn{2}{|c|}{ Prior mental health clinic visits: 0} & Reference & Reference & Reference & Reference & Reference \\
\hline & $1-2$ & $0.91(0.82,1.00)$ & $0.65(0.46,0.91)^{*}$ & $0.95(0.57,1.60)$ & $0.62(0.28,1.35)$ & $0.99(0.37,2.62)$ \\
\hline & $3-4$ & $0.85(0.76,0.94) * *$ & $0.40(0.27,0.59)^{* * *}$ & $0.99(0.59,1.66)$ & $1.01(0.47,2.18)$ & $1.16(0.49,2.76)$ \\
\hline & $5-10$ & $0.77(0.69,0.86)^{* * *}$ & $0.52(0.36,0.74)^{* * *}$ & $0.72(0.42,1.25)$ & $0.68(0.30,1.52)$ & $0.60(0.23,1.57)$ \\
\hline & $\geq 11$ & $0.68(0.59,0.79)^{* * *}$ & $0.46(0.30,0.69)^{* * *}$ & $1.18(0.60,2.33)$ & $0.36(0.12,1.11)$ & $0.69(0.22,2.20)$ \\
\hline \multicolumn{2}{|l|}{ Prior primary care visits } & $1.011(1.003,1.018)^{* *}$ & $1.03(1.01,1.05)^{*}$ & $0.953(0.910,0.998)^{*}$ & $0.98(0.92,1.05)$ & $1.00(0.94,1.05)$ \\
\hline \multicolumn{2}{|l|}{$\geq 1$ psychiatric hospitalization } & $0.73(0.61,0.88)^{* *}$ & $0.89(0.54,1.45)$ & $1.04(0.51,2.11)$ & $1.30(0.38,4.45)$ & $0.93(0.29,2.98)$ \\
\hline \multicolumn{2}{|l|}{ Psychotherapy sessions } & $0.83(0.74,0.94)^{*}$ & $0.86(0.61,1.21)$ & $0.81(0.45,1.46)$ & $0.94(0.36,2.45)$ & $0.41(0.14,1.18)$ \\
\hline \multicolumn{2}{|l|}{ PTSD } & $0.90(0.84,0.98) *$ & $1.29(1.01,1.66)^{*}$ & $0.65(0.44,0.94) *$ & $0.60\left(\begin{array}{lll}0.35 & 1.05)\end{array}\right.$ & $0.72(0.37,1.39)$ \\
\hline
\end{tabular}

$* * * p<0.001 ; * * p<0.01 ; * p<0.05$

PTSD, given the $29 \%$ increase in odds of CAD associated with PTSD in this group. These data cannot confirm whether PTSD preceded the development of CAD. However, given that these patients were 60-96 years of age, their period of military service was likely during the Vietnam War (median age: 60 years), Korean War (median age: 76 years), or World War II (median age: 84 years) [31]. Thus, while it is possible that they were exposed to more recent trauma that promoted the development of PTSD, it seems more likely that their PTSD-related trauma exposure occurred decades earlier while serving during these military eras. Additional research with longitudinal data is needed to assess whether older Black Veterans with depression suffer more cardiovascular consequences from PTSD than other Veterans.

In community samples, Blacks often underutilize medical and mental health services [27, 32, 33], which could contribute to poorer health. The data suggest this is not the case in this VA sample of Black patients. Thus, the relationship between PTSD and higher odds of CAD found in this group does not appear to be due to them having a general underutilization of medical or mental health services. However, we do not have information about the nature of services utilized and whether there were any racial/ethnic differences in treatments received for PTSD.

Interventions that provide social support specifically for this population, such as the group treatment program for Black Veterans with PTSD established at our hospital [34], can reduce PTSD-related social isolation and distress. Such social support also might have stress-buffering effects on cardiovascular health $[35,36]$. In addition, these patients could derive cardiovascular benefits from stress reduction approaches, such as transcendental meditation, which has been associated with lower blood pressure and reduced risk for all-cause mortality, myocardial infarction, and stroke among Blacks adults with CAD [37]. Regarding medical management of Black patients with comorbid depression and PTSD, clinicians should be aware of the potentially increased risk for CAD and may want to focus closer attention on preventative health screens and cardiovascular risk reduction in these patients. Screening for PTSD is standard across all patients in the VA system. It may be beneficial for some non-VA sites to incorporate similar PTSD screenings.

In contrast to the depressed Black patients in this sample, their White and Hispanic counterparts had significantly 
reduced odds of CAD if they had PTSD. The causes of these racial/ethnic differences are uncertain and require further study. Perhaps the finding that Whites with PTSD had reduced odds of CAD was influenced by the accompanying relationships between greater utilization of mental health treatment (e.g., prior year visits to mental health clinics, psychotherapy visits during observation period) and lower CAD. However, the association between PTSD and lower odds of CAD in Hispanic patients was not accompanied by similar links between greater utilization of mental health services and lower odds of CAD.

Although PTSD has been linked to cardiovascular disease and cardiovascular risk factors [38], few studies have examined such relationships among individuals diagnosed with depression and little is known about racial/ethnic variations in such relationships. While one study that utilized medical records reported that PTSD did not predict incident myocardial infarction among depressed Veterans [19], another found that PTSD was associated with self-reported heart disease after adjustment for depression in a sample of young to middle-aged American Indians [39]. Overall, the racial/ ethnic differences in the observed associations between PTSD and CAD in the current study may reflect racial/ ethnic variations in the strength of relationships between PTSD and certain types of cardiovascular disease among individuals with chronic depression.

The prevalence of PTSD in this sample was higher among racial/ethnic minorities than Whites. While this study oversampled for PTSD and is focused on older adults with depression, these racial/ethnic disparities in PTSD are parallel to the findings of other studies showing that PTSD was higher in racial/ethnic minority Veterans than White Veterans $[5,6,8]$. Additional research is needed to understand racial/ethnic disparities in PTSD, not only for the sake of mental health, but also for the sake of physical health, given the increased odds of CAD observed among depressed Black patients with PTSD.

The prevalence of CAD was similar among Hispanics, Asians, and American Indians/Alaskan Natives, roughly 21$23 \%$. While hypertension, diabetes, and hyperlipidemia were significant in the models of CAD among Whites and Blacks, only hypertension reached statistical significance in the models for American Indians/Alaskan Natives and none of these CAD risk factors reached significance in Hispanics or Asians. It is possible that this is due to limitations of the sample sizes for these groups. However, additional research is needed to assess whether the strength of associations between these cardiovascular risk factors and CAD may vary among older adults according to race/ethnicity. For example, while smoking was most prevalent in American Indians/ Alaskan Natives and Blacks, current smoking was not significantly associated with CAD within these groups.

Similar to their White counterparts, Asians had higher odds of CAD if they were married. This seems to be inconsistent with the literature suggesting marriage is beneficial to cardiovascular health [40]. However, our measure of marital status does not convey length of relationship, marriage quality, or marital stress. As with the aforementioned associations between cardiovascular risk factors and $\mathrm{CAD}$, which varied according to race/ethnicity, additional research is needed to assess whether being married differentially confers risks or benefits according to race/ethnicity among older adults with depression.

\section{Strengths}

This research adds to the limited literature available on cardiovascular risk among those with dual psychiatric diagnoses. Given the frequent co-occurrence of mood and anxiety disorders, such research may represent a more realistic representation of certain populations, particularly Veterans. The study adds to the sparse cardiovascular literature on depression or PTSD conducted with older Black, Hispanic, Asian, or American Indian/Alaskan Native samples. Its reliance on medical records that documented diagnoses of CAD, CVD risk factors, PTSD, and depression, rather than relying on selfreports, strengthens the findings. Another strength is the large overall sample size and noteworthy prevalence of diagnosed PTSD, which allowed for detection of differences. The cohort was derived from patients obtaining care from VA medical care at sites throughout the USA, which should enhance generalizability to other VA patients. Utilization of administrative data allowed for adjustment of potentially influential factors that are difficult to account for in other types of studies examining psychiatric disorders for links to CVD, such as documented medical comorbidity (vs. self-report) and accounting for factors of treatment and service utilization.

\section{Limitations}

Though these cross-sectional data from depressed patients provide useful preliminary findings, the relationship between PTSD and CAD in different racial/ethnic groups needs to be further studied with longitudinal data and in samples that do not have depression. Although our overall sample is large, racial/ethnic minorities had notably smaller sample sizes than Whites. However, the proportions of Blacks, Hispanics, Asians, and American Indians/Alaskan Natives in the current sample $(10.7 \%, 4.0 \%, 1.9 \%$, and $1.4 \%$, respectively) are generally consistent with those found in the overall Veteran population $(10.8 \%, 6.0 \%, 1.3 \%$, and $0.6 \%$, respectively) [41], despite the fact that the current sample is specifically examining a sample of older Veterans with depression. Notwithstanding the relatively small sample sizes for minority Veterans, these data help fill a gap in the literature on PTSD and CVD in older Black, Hispanic, Asian, and American Indian/Alaskan Native adults. 
Another potential limitation is the study's over-sampling for PTSD. Although PTSD is more prevalent among Veterans than non-Veterans, it is unclear how well our findings would generalize to non-depressed patients in the VA or to non-VA populations. Further, we had no information on depression or PTSD symptom severity, which could have influenced results. The study also over-sampled women. While the sample sizes of women within most of the racial/ethnic groups were too small for sex/race analyses, sex-stratified logistic regressions conducted on the overall sample (data not shown) indicated that PTSD and CAD were not significantly associated in depressed female patients, but showed an inverse association in their male counterparts (reflecting the pattern in men of all racial/ethnic groups except Black men). However, given the current study's sample limitations, it would be useful to examine PTSD and CAD in a larger racially/ethnically diverse sample of women.

While we accounted for several aspects of psychiatric care, these data cannot specifically account for effectiveness of psychotherapeutic or pharmacologic treatments in treating either depression or PTSD. The study could not assess the relationship of PTSD to CAD in depressed individuals who used non-VA healthcare. Thus, some cases of PTSD and CAD among Veterans may not be captured in these data. The data also are not able to address the issue of potential under-diagnosis of depression or PTSD within the VA. However, the VA healthcare system's performance guidelines and electronic clinical reminders for medical staff to conduct regular screening of both depression and PTSD likely reduces the number of undiagnosed cases of depression and PTSD.

While this study is strengthened by its data being derived from medical records, it is possible that coding for CAD and other study variables could contain errors. In addition, we were able to account for diagnosed obesity, but not BMI, which might have produced different results. The level of diagnosed obesity in the race/ethnicity groups ranged from $11 \%$ to $13 \%$ among these depressed older adults, with the highest prevalence of obesity diagnosis found in White patients $(13 \%)$. A large survey-based study by Nelson [42] reported an obesity rate of $28 \%$ for Veterans (ages 18 and older) receiving VA healthcare and that those over 60 years old had a $25 \%$ increase in odds of obesity (reference group: ages 18 to 39). Based on those findings, it is possible that our diagnosed obesity variable did not capture all individuals with obesity in this sample. Another possible limitation is that the data on smoking were based on self-reports and provided no information about the extent or duration of smoking among current and former smokers. In addition, data on other potential confounders (e.g., diet, exercise) were not available in this dataset. Finally, these data cannot establish temporal ordering or causation between PTSD and CAD among Black patients.

\section{Conclusion}

Results suggest that the relationship between PTSD and CAD varies according to race/ethnicity among older patients with depression. PTSD was associated with an increase in the odds of having $\mathrm{CAD}$ among Black depressed patients. However, it was associated with lower odds of CAD among their White and Hispanic counterparts, while the association did not reach significance among their Asian or American Indian/Alaskan Native counterparts. The findings suggest that Black patients with PTSD and depression may have an elevated burden of CAD.

Acknowledgements This study was funded by the VA Quality Enhancement Research Initiative (QUERI) Rapid Response Project (RRP 10-105). D.C.C. is supported by a VA Health Services Research and Development (HSR\&D) post-doctoral fellowship and by G.E.R.'s Career Scientist Award (RCS 98-353). R.B.T. is supported by a VA HSR\&D Career Development Award (CDA-09-206).

Disclaimer The views expressed in this article are solely those of the authors and do not necessarily reflect the position or policy of the Department of Veterans Affairs.

Conflict of interest Authors Cooper, Trivedi, Nelson, Reiber, Beaver, Eugenio, and Fan declare that they have no conflicts of interest.

Ethical Standards Statement The current work was a study on humans, but it involved no direct experimentation.

\section{References}

1. Ahmadi N, Hajsadeghi F, Mirshkarlo HB, Budoff M, Yehuda R, Ebrahimi R. Post-traumatic stress disorder, coronary atherosclerosis, and mortality. Am J Cardiol. 2011;108(1):29-33.

2. Glaesmer H, Brähler E, Gündel H, Riedel-Heller SG. The association of traumatic experiences and posttraumatic stress disorder with physical morbidity in old age: a German population-based study. Psychosom Med. 2011;73(5):401-6.

3. Pietrzak RH, Goldstein RB, Southwick SM, Grant BF. Physical health conditions associated with posttraumatic stress disorder in U.S. older adults: results from wave 2 of the National Epidemiologic Survey on Alcohol and Related Conditions. J Am Geriatr Soc. 2012;60(2):296-303.

4. Richardson LK, Frueh BC, Acierno R. Prevalence estimates of combat-related post-traumatic stress disorder: critical review. Aust N Z J Psychiatry. 2010;44(1):4-19.

5. Beals J, Manson SM, Shore JH, Friedman M, Ashcraft M, Fairbank JA, et al. The prevalence of posttraumatic stress disorder among American Indian Vietnam veterans: disparities and context. J Trauma Stress. 2002;15(2):89-97.

6. Dohrenwend BP, Turner JB, Turse NA, Lewis-Fernandez R, Yager TJ. War-related posttraumatic stress disorder in Black, Hispanic, and majority White Vietnam veterans: the roles of exposure and vulnerability. J Trauma Stress. 2008;21(2):133-41.

7. Saha S, Freeman M, Toure J, Tippens KM, Weeks C, Ibrahim S. Racial and ethnic disparities in the VA health care system: a systematic review. J Gen Intern Med. 2008;23(5):654-71.

8. Tsai J, Kong G. Mental health of Asian American and Pacific Islander military veterans: brief review of an understudied group. Mil Med. 2012;177(11):1438-44. 
9. Go AS, Mozaffarian D, Roger VL, Benjamin EJ, Berry JD, Borden WB, et al. American Heart Association Statistics Committee and Stroke Statistics Subcommittee. Executive summary: heart disease and stroke statistics - 2013 update: a report from the American Heart Association. Circulation. 2013;127(1):143-52.

10. Villa VM, Harada ND, Washington D, Damron-Rodriguez J. The health and functional status of US veterans aged 65+: implications for VA health programs serving an elderly, diverse veteran population. Am J Med Qual. 2003;18(3):108-16.

11. Ginzburg K, Ein-Dor T, Solomon Z. Comorbidity of posttraumatic stress disorder, anxiety and depression: a 20-year longitudinal study of war veterans. J Affect Disord. 2010;123(1-3):249-57.

12. Kinder LS, Bradley KA, Katon WJ, Ludman E, McDonell MB, Bryson CL. Depression, posttraumatic stress disorder, and mortality. Psychosom Med. 2008;70(1):20-6.

13. Rytwinski NK, Scur MD, Feeny NC, Youngstrom EA. The cooccurrence of major depressive disorder among individuals with posttraumatic stress disorder: a meta-analysis. J Trauma Stress. 2013;26(3):299-309.

14. Lett HS, Blumenthal JA, Babyak MA, Sherwood A, Strauman T, Robins $\mathrm{C}$, et al. Depression as a risk factor for coronary artery disease: evidence, mechanisms, and treatment. Psychosom Med. 2004;66(3):305-15

15. Zellweger MJ, Osterwalder RH, Langewitz W, Pfisterer ME. Coronary artery disease and depression. Eur Heart J. 2004;25(1):3-9.

16. Hankin CS, Spiro A, Miller DR, Kazis L. Mental disorders and mental health treatment among US Department of Veterans Affairs outpatients: the veterans health study. Am J Psychiatry. 1999;156: 1924-30.

17. Spitzer C, Barnow S, Völzke H, John U, Freyberger HJ, Grabe HJ. Trauma, posttraumatic stress disorder, and physical illness: findings from the general population. Psychosom Med. 2009;71(9):1012-7.

18. Shankman SA, Nadelson J, McGowan SK, Sovari AA, Vidovich MI. The predictive power of depression screening procedures for veterans with coronary artery disease. Vasc Health Risk Manag. 2012;8:233-8.

19. Scherrer JF, Chrusciel T, Zeringue A, Garfield LD, Hauptman PJ, Lustman PJ, et al. Anxiety disorders increase risk for incident myocardial infarction in depressed and nondepressed Veterans Administration patients. Am Heart J. 2010;159(5):772-9.

20. Quiñones AR, Thielke SM, Beaver KA, Trivedi RB, Williams EC, Fan VS. Racial and ethnic differences in the receipt of antidepressants and psychotherapy among veterans with depression. Psychiatr Serv. 2013 Nov 1.

21. Veterans Health Administration Office of Quality and Performance. Executive Career Field Network Director Performance Measurement System and JCAHO Hospital Core Measures (Technical Manual). Washington: Veterans Health Administration; 2007.

22. Maynard C, Chapko MK. Data resources in the Department of Veterans Affairs. Diabetes Care. 2004;27 Suppl 2:B22-6.

23. Kashner TM. Agreement between administrative files and written medical records: a case of the Department of Veterans Affairs. Med Care. 1998;36(9):1324-36.

24. Deyo RA, Cherkin DC, Ciol MA. Adapting a clinical comorbidity index for use with ICD-9-CM administrative databases. J Clin Epidemiol. 1992;45:613-9.

25. Bradley KA, Williams EC, Achtmeyer CE, Volpp B, Collins BJ, Kivlahan DR. Implementation of evidence-based alcohol screening in the Veterans Health Administration. Am J Manage Care. 2006;12(10):597-606.
26. Bush K, Kivlahan DR, McDonell MB, Fihn SD, Bradley KA. The AUDIT alcohol consumption questions (AUDIT-C): an effective brief screening test for problem drinking. Ambulatory Care Quality Improvement Project (ACQUIP). Alcohol Use Disorders Identification Test. Arch Intern Med. 1998;158(16): 1789-95.

27. Burgess DJ, Ding Y, Hargreaves M, van Ryn M, Phelan S. The association between perceived discrimination and underutilization of needed medical and mental health care in a multi-ethnic community sample. J Health Care Poor Underserved. 2008;19(3):894-911.

28. Boswell JF, McAleavey AA, Castonguay LG, Hayes JA, Locke BD. Previous mental health service utilization and change in clients' depressive symptoms. J Couns Psychol. 2012;59(3):368-78.

29. Scherrer JF, Garfield LD, Lustman PJ, Hauptman PJ, Chrusciel T, Zeringue A, et al. Antidepressant drug compliance: reduced risk of MI and mortality in depressed patients. Am J Med. 2011;124(4): $318-24$.

30. Whittle J, Conigliaro J, Good CB, Hanusa BH, Macpherson DS. Black-white differences in severity of coronary artery disease among individuals with acute coronary syndromes. J Gen Intern Med. 2002;17(11):867-73.

31. U.S. Department of Veterans Affairs. VA Rocky Mountain Network 2007 Annual Report. Washington: VA Rocky Mountain Network; 2007.

32. Gaskin DJ, Dinwiddie GY, Chan KS, McCleary R. Residential segregation and disparities in health care services utilization. Med Care Res Rev. 2012;69(2):158-75.

33. Mayberry RM, Mili F, Ofili E. Racial and ethnic differences in access to medical care. Med Care Res Rev. 2000;57 Suppl 1:108-45.

34. Jones L, Brazel D, Peskind ER, Morelli T, Raskind MA. Group therapy program for African-American veterans with posttraumatic stress disorder. Psychiatr Serv. 2000;51(9):1177-9.

35. Bowen KS, Uchino BN, Birmingham W, Carlisle M, Smith TW, Light KC. The stress-buffering effects of functional social support on ambulatory blood pressure. Health Psychol. 2013 Nov 18.

36. Lett HS, Blumenthal JA, Babyak MA, Strauman TJ, Robins C, Sherwood A. Social support and coronary heart disease: epidemiologic evidence and implications for treatment. Psychosom Med. 2005;67(6):869-78.

37. Schneider RH, Grim CE, Rainforth MV, Kotchen T, Nidich SI, Gaylord-King C, et al. Stress reduction in the secondary prevention of cardiovascular disease: randomized, controlled trial of transcendental meditation and health education in Blacks. Circ Cardiovasc Qual Outcomes. 2012;5(6):750-8.

38. Dedert EA, Calhoun PS, Watkins LL, Sherwood A, Beckham JC. Posttraumatic stress disorder, cardiovascular and metabolic disease: a review of the evidence. Ann Behav Med. 2010;39:61-78.

39. Sawchuk CN, Roy-Byrne P, Goldberg J, Manson S, Noonan C, Beals $\mathrm{J}$, et al. The relationship between post-traumatic stress disorder, depression and cardiovascular disease in an American Indian tribe. Psychol Med. 2005;35(12):1785-94.

40. Baune BT, Stuart M, Gilmour A, Wersching H, Arolt V, Berger K. Moderators of the relationship between depression and cardiovascular disorders: a systematic review. Gen Hosp Psychiatry. 2012;34(5): 478-92.

41. U.S. Department of Veterans Affairs. Minority veterans: 2011. Washington: U.S. Government Printing Office; 2013.

42. Nelson KM. The burden of obesity among a national probability sample of veterans. J Gen Intern Med. 2006;21(9):915-9. 